\title{
Solar motion and discharge of Paraná River, South America: Evidence for a link
}

\author{
A. Antico ${ }^{1}$ and D. M. Kröhling ${ }^{1}$ \\ Received 9 July 2011; revised 9 September 2011; accepted 14 September 2011; published 13 October 2011.
}

[1] A link between climate and the motion of the Sun around the barycenter of the solar system has been suggested, mostly on the basis of interannual cycles observed in climatological data from the Northern Hemisphere. This study provides empirical evidence for a connection between this solar motion and discharge of the Paraná River, which has the second largest drainage basin of South America. Solar motion is described here by the absolute value of the time derivative of solar angular momentum $(|\tau|)$. For the time period 1904-2008, the time evolution of both $|\tau|$ and river discharge is dominated by cycles with periods between 7 and 9 yr. Within this frequency band, a statistically significant anticorrelation is found between $|\tau|$ and river discharge with minima of $|\tau|$ coinciding with increased river discharge. Citation: Antico, A., and D. M. Kröhling (2011), Solar motion and discharge of Paraná River, South America: Evidence for a link, Geophys. Res. Lett., 38, L19401, doi:10.1029/ 2011GL048851.

\section{Introduction}

[2] On a global and long-term basis, the Sun is the primary energy source of the climate system. However, the extent to which the Sun contributes to climate change and some of the possible response mechanisms are controversial [Versteegh, 2005; Almasi and Bond, 2009]. Nonetheless, there is empirical evidence for links between climate and solar phenomena such as the motion of the Sun around the barycenter of the planetary system (hereafter referred as to solar motion), which is mainly driven by gravitational forces between the Sun and jovian planets [e.g., Landscheidt, 1988, 2000; Charvátová and Střeštik, 2004; Tomasino et al., 2004; Zanchettin et al., 2008; Scafetta, 2010]. Most of this evidence is commonly observed in the Northern Hemisphere probably because instrumental, historical and geological data are more available in this hemisphere than in the Southern Hemisphere (SH), and not because of the absence of solar-driven climate changes in the SH [Versteegh, 2005]. This study contributes to fill this information gap by showing evidence for a relationship between near-decadal discharge cycles in a large South American river and solar motion.

[3] The hypothesis that solar motion influence climate indirectly by driving solar variability had been proposed [e.g., Landscheidt, 1988, 2000; Charvátová and Střeštik, 2004]. Nevertheless, results of recent solar physics studies indicate that this hypothesis can be rejected [e.g., de Jager and Versteegh, 2005; Versteegh, 2005]. Alternatively, it was suggested that

\footnotetext{
${ }^{1}$ CONICET, Facultad de Ingeniería y Ciencias Hídricas, Universidad Nacional de Litoral, Santa Fe, Argentina.

Copyright 2011 by the American Geophysical Union. 0094-8276/11/2011GL048851
}

small changes in the Earth-Sun distance associated with solar motion could influence climate directly by modifying luni-solar tidal forces and the solar radiation received by the Earth [Mackey, 2007; Scafetta, 2010]. In the climate system, the effect of these weak forcings would be amplified by feedback processes, resonance, and collective synchronization of oscillators. These explanations, however, remain speculative because the underlying physical mechanisms are unknown. Hence, finding new empirical evidence for climate-solar motion links may contribute to elucidate response mechanisms.

[4] The basin of the Paraná River, the second largest river basin of South America, is an appropriate region for searching evidence of Sun-climate connections. One reason for this is that the regional scale of the Paraná basin can facilitate the identification of climatic responses to solar changes [Versteegh, 2005]. At the global scale, a Sun-climate link would not be found because the climate responses to a solar forcing may have different signs in different regions and, therefore, regional climate changes would tend to cancel each other in the computation of global means. At subregional scales, climate variability could be mainly influenced by local processes not related to the Sun and, consequently, a climatic signal of solar origin would be hard to detect. Also, time series of Paraná River discharge could be more suitable than precipitation records for finding low-frequency (e.g., interannual) climate variability driven by the Sun. This is because, when precipitation is integrated over a river basin, the water storage capacity of the basin can damp (leave unchanged) the high-frequency (lowfrequency) cycles of precipitation, i.e., the water storage capacity can act as a lowpass filter [Milly and Wetherald, 2002].

[5] Time series of Paraná River discharge are dominated by a long-term trend (oscillations with periods greater than $40 \mathrm{yr}$ ) and near-decadal cycles (periods between 7 and $9 \mathrm{yr}$ ) [Robertson and Mechoso, 1998; Pasquini and Depetris, 2006; Krepper et al., 2008]. Anthropogenic activities (e.g., deforestation) and a possible role of internal climate oscillations (e.g., the North Atlantic Oscillation) had been invoked to interpret this discharge variability [e.g., Robertson and Mechoso, 1998; Krepper et al., 2008]. However, to the best of our knowledge, the near-decadal cycles of Paraná River discharge have not yet been related to solar cycles. It is noted that, although Mauas et al. [2011] found a possible link between Paraná River discharge and solar activity, they did not analyze near-decadal hydrological variability since they only considered oscillations with periods between 11 and $50 \mathrm{yr}$.

[6] Interestingly, 7-9-yr discharge cycles are present not only in the Paraná River but also in the Po River (Northern Italy). The variability of winter Po River discharge is characterized by a cycle with a period of approximately $8.7 \mathrm{yr}$ [Tomasino et al., 2004]. Previous studies lended support to a relationship between solar motion and the 8.7-yr discharge cycle of the Po River [e.g., Landscheidt, 2000; Tomasino 


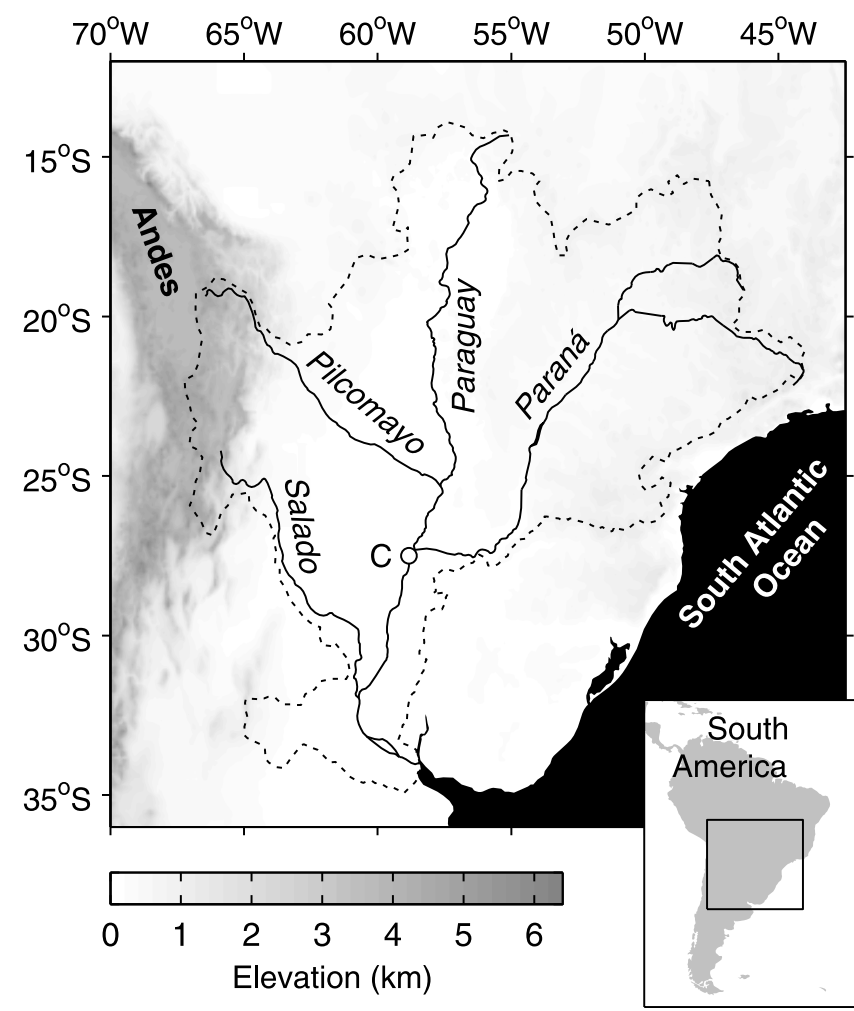

Figure 1. Map showing the Paraná River system (black lines) and its drainage basin (delimited by the dashed line). Names of main rivers are given in italics and terrain elevation is depicted as a gray scale map. The open circle indicates the position of the gauging station at Corrientes city (C), which is located south of the confluence of Paraguay and Paraná Rivers.

et al., 2004]. Hence, we propose that cycles of Paraná River discharge with periods between 7 and 9 yr could also be related to solar motion. To test this hypothesis, we compare time series of the following variables in the time and frequency domains: (i) absolute value of the time derivative of solar angular momentum, a measure of solar motion, and (ii) Paraná River discharge.

\section{Data and Methods}

[7] The Paraná River drains an area of about $2.6 \times 10^{6} \mathrm{~km}^{2}$ in southeastern South America (Figure 1). For the period 1904-2008, time series of annual-mean Paraná River discharge $(D)$ from the Corrientes gauging station are analysed in this study. Note that because of the location of this station, discharge estimated at this site reflects precipitation integrated over most of the basin (see station location in Figure 1). South of this station, there are two other gauging stations at the cities of Paraná and Timbúes where Paraná River discharge has been measured since 1904 and 1905, respectively. A comparison between time series of $D$ from these two stations (data not shown) and from Corrientes station (data shown in section 3 ) reveals that these three time series have very similar variability. Thus, the conclusions of the present study would not change if river discharge data from Paraná or Timbúes station would be used instead.

[8] To characterize the motion of the Sun around the center of mass of the solar system (CM), the annual-mean absolute value of the time derivative of solar angular momentum $(|\tau|)$ is considered (i.e., the annual-mean absolute value of solar torque is considered). The two-step estimation of $|\tau|$ is described as follows. First, the position and velocity of the center of the Sun (CS) with respect to $\mathrm{CM}$ are computed at the beginning of calendar months according to Giorgini et al. [1996] and used to obtain the solar angular momentum $(L)$ (see positions of CS on the ecliptic plane and the component of $L$ perpendicular to this plane in Figure 2 (top and middle)). Second, absolute values
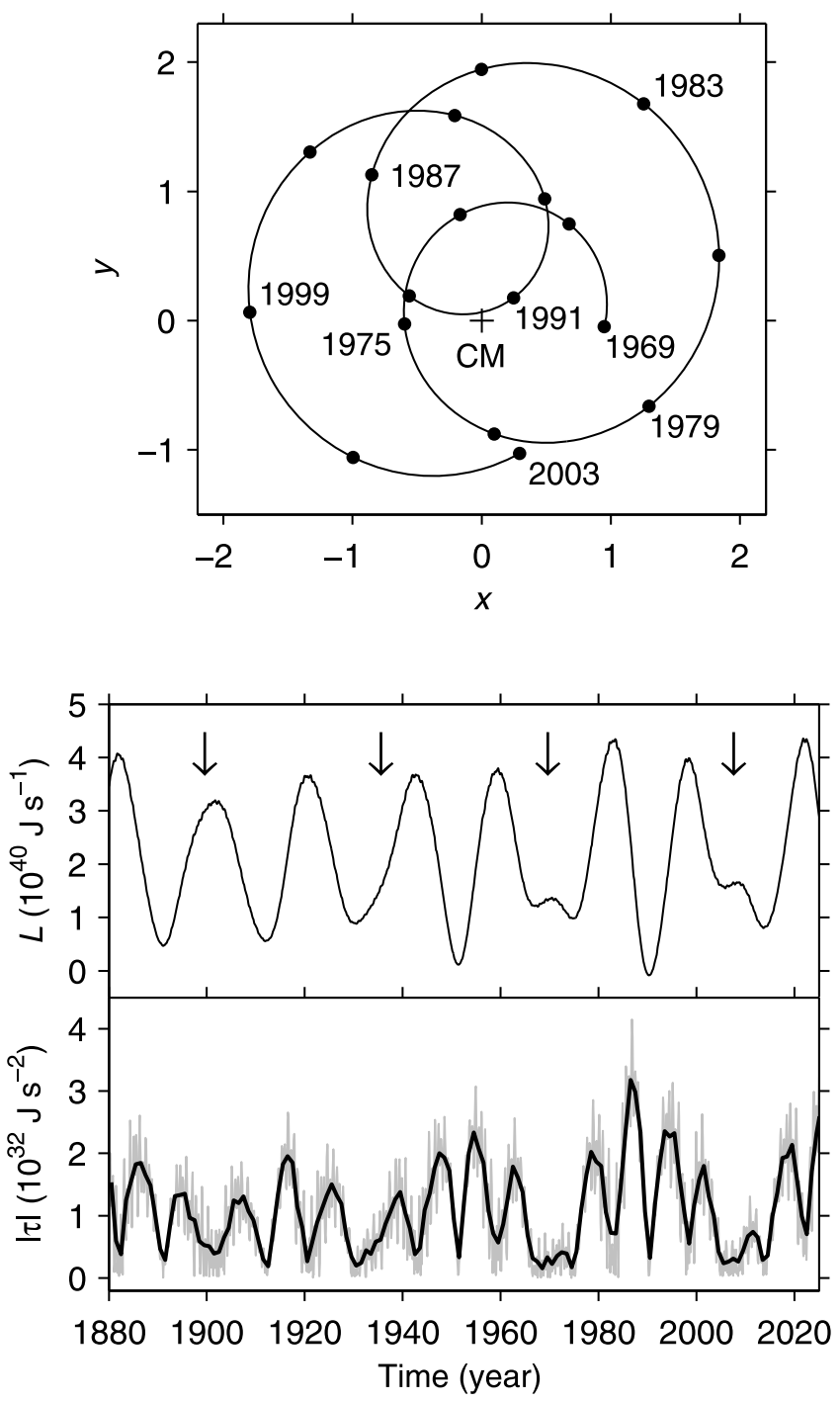

Figure 2. (top) Trajectory of the Sun's center around the center of mass of the Solar system (CM) for the period 1969-2003 (dots are every 2 yr and correspond to the beginning of calendar years); distances $x$ and $y$, on the ecliptic plane, are expressed as fraction of Sun's radius (photosphere radius). Monthly values of (middle) component of the solar angular momentum perpendicular to the ecliptic plane $(L)$ and (bottom, gray line) solar torque magnitude $(|\tau|)$ for the period 1880-2025; annual means of $|\tau|$ are also shown (bottom, black line). This torque is defined as the time derivative of the angular momentum of the Sun's motion around CM. Arrows indicate perturbations in the sinusoidal shape of the time series of $L$, which occurred nearly every $36 \mathrm{yr}$. 


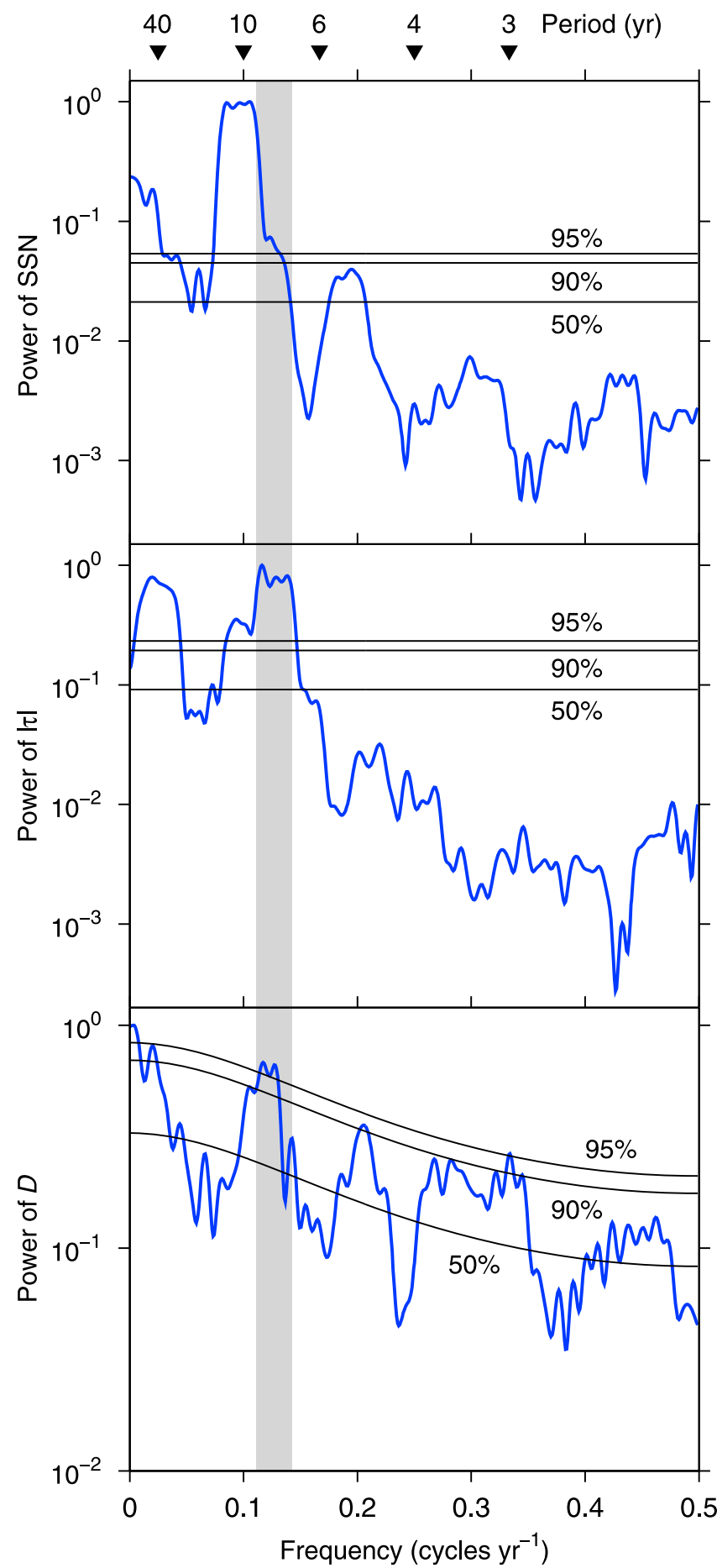

Figure 3. Multitaper power spectra (three tapers) of annual means of (top) sunspot number (SSN), (middle) magnitude of solar torque $(|\tau|)$, and (bottom) Paraná River discharge $(D)$ for the period 1904-2008. In all panels, power is normalized by its maximum value. The $50 \%, 90 \%$ and $95 \%$ significant levels for a red-noise (white-noise) process are shown in the bottom (top and middle). Above the top panel, filled triangles indicate oscillation periods. The shaded region corresponds to periods between 7 and $9 \mathrm{yr}$.

of the time derivative of $L$ are obtained at the beginning of every calendar month and then used to obtain annual means of $|\tau|$ (Figure 2, bottom).
[9] To compare time series of annual-mean $|\tau|$ and $D$ in the frequency domain, the multitaper method of spectral estimation is used [Ghil et al., 2002]. The statistical significance of spectral peaks is determined by testing a white-noise hypothesis in the spectra of solar variables [Burroughs, 2003], and by testing a red-noise hypothesis in the spectrum of $D$ [Ghil et al., 2002]. To facilitate the comparison of time series of $|\tau|$ and $D$ in the time domain, a finite impulse response filter is implemented to pass the frequency bands containing statistically significant spectral peaks.

\section{Results and Discussion}

[10] Time series of annual-mean $|\tau|$ and $D$ are both dominated by cycles with periods between 7 and $9 \mathrm{yr}$, which are statistically significant at the $95 \%$ level in the spectra of these variables (Figure 3). Peaks within this frequency band are also found when the maximum entropy spectral analysis [Ghil et al., 2002] is performed on time series of $|\tau|$ and $D$ (results of this analysis are not shown). Previous studies also reported significant cycles of Paraná River discharge with periods between 7 and 9 yr [e.g., Robertson and Mechoso, 1998; Pasquini and Depetris, 2006; Krepper et al., 2008]. The fact that we obtained significant spectral peaks of $|\tau|$ and $D$ at the same frequencies lends support to a link between solar motion and river discharge. These frequencies are higher than those corresponding to cycles of sunspot number (SSN), a popular index of solar activity (Figure 3 ). This suggests that, at neardecadal timescales, there is no direct relationship between solar irradiance variability and river discharge.

[11] To reconstruct the near-decadal cycles of $|\tau|$ and $D$, a bandpass filter with cut-off periods of 7 and $9 \mathrm{yr}$ is applied to time series of these variables (Figure 4). The Pearson's coefficient of zero-lag correlation $\left(r_{0}\right)$ between filtered time series of $|\tau|$ and $D$ is -0.63 (i.e., minima of $|\tau|$ tend to coincide with maxima of $D$ ). This value is statistically significant since the $99 \%$ level estimated for the 105-element time series of $D$ is $-2.58 / \sqrt{103}=-0.25$ [Panofsky and Brier, 1958]. This high significance is corroborated by a bias corrected and accelerated percentile bootstrap method [DiCicio and Efron, 1996] which give a 99\% confidence interval for $r_{0}$ with an upper limit equal to $-0.43(10,000$ bootstrap data samples were generated). Also, the absolute value of $r_{0}$ is greater than that of lagged correlation coefficients obtained by applying lags of 1 and $2 \mathrm{yr}$ to the time series of $D$. This is consistent with an immediate response of the hydrological cycle to solar motion and, therefore, could reflect the influence of solar motion on fast-response elements of the Earth system (e.g., atmosphere and the ocean surface layer). Conversely, slow-response elements (e.g., deep ocean) would not be influenced.

[12] Of twelve well-marked minima of filtered times series of $|\tau|$, ten approximately correspond to maxima of filtered time series of $D$ (compare thick lines in Figure 4). This correspondence between dominant cycles of $|\tau|$ and $D$ is remarkable if we consider that, at near-decadal timescales, $D$ could respond not only to $|\tau|$ changes, but also to other natural forcings of climate (e.g., geomagnetism, tides and volcanism) and internal climate oscillations (e.g., El Niño Southern Oscillation and the North Atlantic Oscillation (NAO)). Indeed, Robertson and Mechoso [1998] proposed a possible link between the NAO and near-decadal cycles of Paraná River discharge. Nevertheless, this does not 


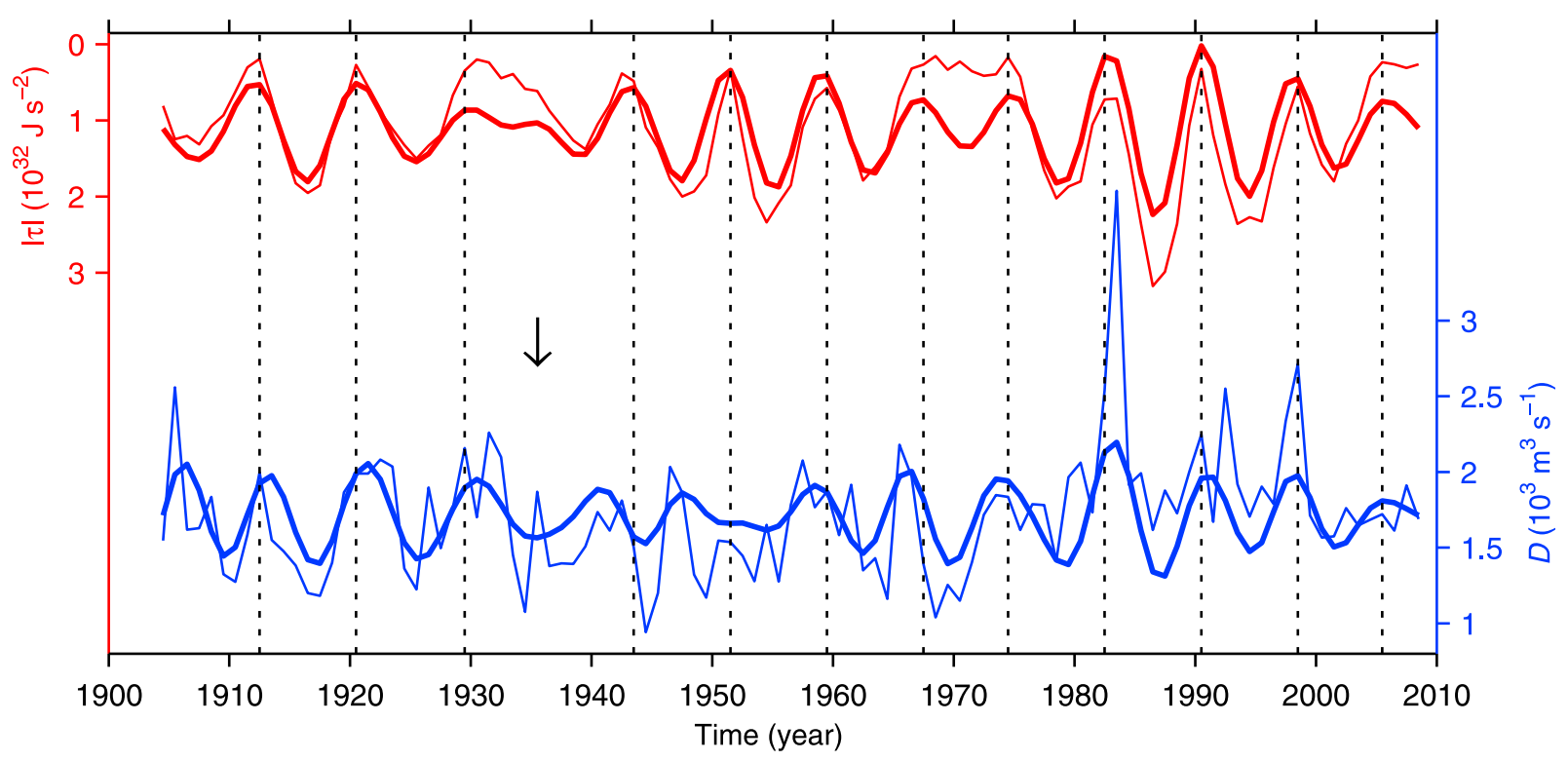

Figure 4. Annual means of magnitude of solar torque $(|\tau|)$ and Paraná River discharge $(D)$ for the period 1904-2008. Note that the vertical axis for $|\tau|$ is reversed. Smoothed curves (thick lines) are obtained by applying a bandpass filter with lower and upper cut-off frequencies of $1 / 9$ and $1 / 7$ cycles $\mathrm{yr}^{-1}$, respectively, to the time series of annual-mean $|\tau|$ and $D$ (thin lines); a 15th-order finite impulse response filter is used. Vertical dashed lines correspond to well-marked minima of $|\tau|$. The arrow indicates the perturbation in the sinusoidal shape of solar angular momentum that occurred around 1935 .

necessarily exclude the possible effect of solar motion on river discharge since connections between the NAO and solar motion were already suggested [Zanchettin et al., 2008; Scafetta, 2010]. If these connections are accepted, the NAO could be viewed as an element of the physical mechanism of the response of river discharge to solar motion. Alternatively, both the NAO and river discharge could respond independently to solar motion.

[13] The relationship between $|\tau|$ and $D$ found in this study for the period prior to 1935 was also observed in the Po River basin [Landscheidt, 2000; Tomasino et al., 2004]. Around 1935, a phase reversal occurred and, after this year, minima of $|\tau|$ approximately coincide with low values of the Po River discharge. Landscheidt [2000] suggested that this reversal could be associated with a perturbation in the sinusoidal shape of solar angular momentum that occurred around 1935 (see middle panel of Figure 2). Interestingly, the only two successive $|\tau|$ minima that do not coincide with maxima of Paraná river discharge occurred between 1935 and 1955 (see Figure 4). Hence, the aforementioned perturbation of solar angular momentum could be also responsible for a change in the response of Paraná River discharge to solar motion during the 1935-1955 period. It is also possible that, during this period, near-decadal variability of river discharge was mainly driven by forcings not related to solar motion, which were mentioned earlier in this section. Moreover, the absence of a climate-solar motion link would be associated with a weak solar forcing [Versteegh, 2005]. Note that peak-to-trough changes in near-decadal cycles of $|\tau|$ are small between 1930 and 1940 (see upper thick line in Figure 4).

\section{Conclusions}

[14] For the period 1904-2008, annual means of the absolute value of solar torque $(|\tau|)$ and discharge of the Paraná River $(D)$, which has the second largest river basin of South America, are compared in the time and frequency domains. This torque is defined as the time derivative of the angular momentum associated with the motion of the Sun around the barycenter of the solar system.

[15] Time series of $|\tau|$ and $D$ are both dominated by cycles with periods between 7 and $9 \mathrm{yr}$. Within this frequency band, a statistically significant anticorrelation is found between these two variables with minima of $|\tau|$ coinciding with maxima of $D$ (the Pearson's correlation coefficient is -0.63). This anticorrelation between dominant cycles of $|\tau|$ and $D$ is remarkable if we consider that river discharge variability could be driven not only by solar motion but also by other forcings and internal climate oscillations.

[16] Finally, it is important to note that climate data from the Northern Hemisphere was used in most of the previous studies that already revealed evidence for links between climate and solar motion. Hence, our novel results for South America lend support to the hypothesis that solar motion could affect regional climate worldwide. Therefore, we hope that this letter will motivate future studies aimed to test this hypothesis by obtaining more empirical evidence for relationships between climate and solar motion, and by developing theories of possible response mechanisms. To accept this hypothesis would have profound implications for climate predictability because of the deterministic nature of solar motion, i.e., forecasting capabilities would be improved.

[17] Acknowledgments. The authors thank two anonymous reviewers for their constructive comments that improved the manuscript. We thank the Subsecretaría de Recursos Hídricos of Argentina for providing Paraná River discharge data. The spectral analysis algorithms were provided by the SSAMTM group at UCLA (http://www.atmos.ucla.edu/tcd/ssa/). The FIR filter algorithm was provided by the Ocean Time Series group at SIO (http://mooring.ucsd edu/). Sunspot number data were provided by STPD-NGDC-NOAA (http:// www.ngdc.noaa.gov/stp/). Five-minute resolution terrain elevation data were provided by RDA-CISL-NCAR (http://dss.ucar.edu/). This work was supported by the PIRHCa and PRODACT programs at FICH-UNL, Argentina. 
[18] The Editor thanks Gerard Versteegh and an anonymous reviewer for assistance in evaluating this paper.

\section{References}

Almasi, P. F., and G. C. Bond (2009), Sun-climate connections, in Encyclopedia of Paleoclimatology and Ancient Environments, edited by V. Gornitz, pp. 929-935, Springer, The Netherlands.

Burroughs, W. J. (2003), Extraterrestrial influences, in Weather Cycles: Real or Imaginary?, pp. 173-210, Cambridge Univ. Press, New York.

Charvátová, I., and J. Stř̌eštik (2004), Periodicities between 6 and 16 years in surface air temperature in possible relation to solar inertial motion, J. Atmos. Sol. Terr. Phys., 66, 219-227.

de Jager, C., and J. M. Versteegh (2005), Do planetary motions drive solar variability?, Sol. Phys., 229, 175-179, doi:10.1007/s11207-005-4086-7.

DiCicio, T. J., and B. Efron (1996), Bootstrap confidence intervals, Stat. Sci., 11, 189-228.

Ghil, M., et al. (2002), Advanced spectral methods for climatic time series, Rev. Geophys., 40(1), 1003, doi:10.1029/2000RG000092.

Giorgini, J. D., D. K. Yeomans, A. B. Chamberlin, P. W. Chodas, R. A. Jacobson, M. S. Keesey, J. H. Lieske, S. J. Ostro, E. M. Standish, and R. N. Wimberly (1996), JPL's on-line solar system data service, Bull. Am. Astron. Soc., 28, 1158. (Available at http://ssd.jpl.nasa.gov/?horizons.)

Krepper, C. M., N. O. García, and P. D. Jones (2008), Low-frequency response of the upper Paraná basin, Int. J. Climatol., 28, 351-360.

Mackey, R. (2007), Rhodes Fairbridge and the idea that the solar system regulates the Earth's climate, J. Coastal Res., 50, 955-968.

Milly, P. C. D., and R. T. Wetherald (2002), Macroscale water fluxes: 3. Effects of land processes on variability of monthly river discharge, Water Resour. Res., 38(11), 1235, doi:10.1029/2001WR000761.
Landscheidt, T. (1988), Solar rotation, impulses of the torque in the Sun's motion, and climatic variation, Clim. Change, 12, 265-295.

Landscheidt, T. (2000), River Po discharges and cycles of solar activity, Hydrol. Sci. J., 45, 491-493.

Mauas, P. J. D., A. P. Buccino, and E. Flamenco (2011), Long-term solar activity influences on South American rivers, J. Atmos. Sol. Terr. Phys., 73, 377-382, doi:10.1016/j.jastp.2010.02.019.

Panofsky, H. A., and G. W. Brier (1958), Some Applications of Statistics to Meteorology, Penn. State Univ. Press, 224 pp., State College.

Pasquini, A., and P. J. Depetris (2006), Discharge trends and flow dynamics of South American rivers draining the southern Atlantic seabord: An overview. J. Hydrol., 333, 385-399.

Robertson, A. W., and C. R. Mechoso (1998), Interannual and decadal cycles in river flows of southeastern South America, J. Clim., 11, 2570-2581.

Scafetta, N. (2010), Empirical evidence for a celestial origin of the climate oscillations and its implications, J. Atmos. Sol. Terr. Phys., 72, 951-970, doi:10.1016/j.jastp.2010.04.015.

Tomasino, M., D. Zanchettin, and P. Traverso (2004), Long-range forecasts of River Po discharges based on predictable solar activity and a fuzzy neural network model, Hydrol. Sci. J., 49, 673-684.

Versteegh, G. (2005), Solar forcing of climate. 2: Evidence from the past, Space Sci. Rev., 120, 243-286, doi:10.1007/s11214-005-7047-4.

Zanchettin, D., A. Rubino, P. Traverso, and M. Tomasino (2008), Impact of variations in solar activity on hydrological decadal patterns in northern Italy, J. Geophys. Res., 113, D12102, doi:10.1029/2007JD009157.

A. Antico and D. M. Kröhling, CONICET, Facultad de Ingeniería y Ciencias Hídricas (FICH), Universidad Nacional de Litoral, C.C. 217 (3000), Santa Fe, Argentina. (aantico@santafe-conicet.gov.ar) 